\title{
Altura do joelho como medida alternativa confiável na avaliação nutricional de idosos
}

\author{
Knee-height as a reliable alternative \\ measure for nutritional assessment \\ in older adults
}

Vera Elizabeth CLOSS 1

Ana Maria Pandolfo FEOLI²

Carla Helena Augustin SCHWANKE ${ }^{1}$

\section{R E S U M O}

\section{Objetivo}

Avaliar o uso da altura do joelho como medida alternativa confiável na avaliação nutricional de idosos atendidos em um ambulatório de geriatria.

\section{Métodos}

Estudo transversal com idosos atendidos em ambulatório de geriatria de um hospital universitário, entre novembro de 2009 e novembro de 2010. Variáveis investigadas: gênero, idade, peso, estatura, altura do joelho, estatura estimada através das equações de Chumlea, índice de massa corporal para as medidas de estatura aferida e estimada e classificado segundo Lipschitz, e dados sociodemográficos. Na análise descritiva dos dados foram usadas medidas de tendência central, dispersão e proporção; a homogeneidade entre as medidas foi verificada através do coeficiente de correlação intraclasse e do gráfico de Bland e Altman e a concordância entre a avaliação nutricional baseada no índice de massa corporal calculado a partir da estatura aferida e a avaliação nutricional baseada no índice de massa corporal calculado a partir da estatura estimada foi avaliada através do coeficiente Kappa ponderado com pesos quadráticos.

\section{Resultados}

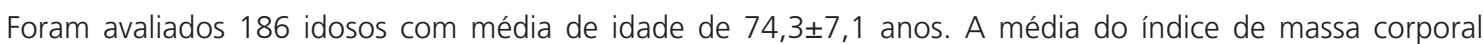
aferido e estimado foi $28,96 \pm 5,86 \mathrm{~kg} / \mathrm{m}^{2}$ e $27,95 \pm 5,47 \mathrm{~kg} / \mathrm{m}^{2}$, respectivamente, e a prevalência de sobrepeso

\footnotetext{
1 Pontifícia Universidade Católica do Rio Grande do Sul, Instituto de Geriatria e Gerontologia, Programa de Pós-Graduação em Gerontologia Biomédica. Av. Ipiranga, 6681, Prédio 81, $7^{\circ}$ andar, sala 703, 90619-900, Porto Alegre, RS, Brasil. Correspondência para/Correspondence to: CHA SCHWANKE. E-mail: <schwanke@pucrs.br>.

2 Pontifícia Universidade Católica do Rio Grande do Sul, Faculdade de Enfermagem, Nutrição e Fisioterapia, Curso de Nutrição. Porto Alegre, RS, Brasil.
} 
foi de $59,7 \%$ para o índice de massa corporal aferido e 53,2\% para o estimado. Foi observada uma boa concordância (Kappa=0,79; $p<0,001)$ entre as duas avaliações.

\section{Conclusão}

Os resultados mostraram ser possível utilizar a altura do joelho na estimativa da estatura para cálculo do índice de massa corporal em idosos atendidos em ambulatório, alternativa útil para aqueles que apresentam limitações na aferição das medidas, sendo esta uma medida alternativa importante na avaliação nutricional de idosos.

Palavras-chave: Antropometria. Avaliação nutricional. Estatura. Idoso. Técnicas de estimativa.

\section{A B S T R A C T}

\section{Objective}

To evaluate the use of knee-height as a reliable alternative measure for nutritional evaluation in older adults in a geriatrics outpatient clinic.

\section{Methods}

Cross-sectional study with elderly patients being treated in a geriatrics outpatient clinic of a University Hospital between November 2009 and November 2010. Variables investigated: gender, age, weight, stature, kneeheight, estimated stature using Chumlea equations, body mass index based on measured and estimated stature and classified according to Lipschitz, and socio-demographic characteristics. Measures of central tendency, dispersion, and proportion were used in the descriptive data analysis. Homogeneity between these measures was verified using intraclass correlation coefficient and Bland and Altman plot, and the correlation between the nutritional assessment concerning the body mass index calculated based on the measured stature and the nutritional assessment calculated based on the estimated stature was established using the quadratic weighted Kappa coefficient.

\section{Results}

One hundred eight-six elderly patients with average age of $74.3 \pm 7.1$ years were evaluated. The average body mass index calculated and estimated were, respectively, 28.96 $\pm 5.86 \mathrm{kgm}^{2}, 27.95 \pm 5.47 \mathrm{kgm}^{2}$, and the prevalence of overweight was $59.7 \%$ for body mass index calculated and $53.2 \%$ for body mass index estimated. A good agreement between the evaluations was observed (Kappa=0.79; $p<0.001$ ).

\section{Conclusion}

Results showed that it is possible to use the knee-height for the estimation of stature to determine the body mass index in elderly patients in a geriatric outpatient clinic. It proved a useful alternative especially when it is difficult to obtain accurate measures in older adults, suggesting that knee-height is an important alternative measure for the nutritional status evaluation in elderly patients.

Keywords: Anthropometry. Nutritional assessment. Body height. Aged. Estimation techniques.

\section{N T R O D U Ç Ã O}

A estatura é um indicador amplamente utilizado na avaliação clínica de indivíduos. Trata-se de uma medida antropométrica que pode ser utilizada isoladamente e em equações, entre elas a de obtenção do Índice de Massa Corporal (IMC), ferramenta de uso reconhecido na determinação do estado nutricional' ${ }^{1}$.

Medir a estatura em idosos, entretanto, impõe dificuldades, porque, muitas vezes, eles não conseguem ficar eretos para a medição, devi- do a problemas osteoarticulares, tais como achatamento dos espaços intervertebrais, osteoporose, cifose dorsal, arqueamento dos membros inferiores e do arco plantar². Essas mudanças, associadas ao envelhecimento, promovem alterações que podem contribuir para redução da estatura ${ }^{2-4}$. Além disso, fragilidade, instabilidade postural, síndrome da mobilidade, casos de amputação ou mesmo confinamento ao leito são situações que podem dificultar a obtenção da medida ${ }^{5,6}$. Por consequência, desconsiderar o fato de que a estatura aferida do idoso pode não mais 
refletir a medida máxima alcançada durante a vida pode ter implicações sobre a interpretação do IMC por subestimar seu resultado?.

Assim, esforços têm sido realizados no desenvolvimento de equações estimativas de estatura ${ }^{4}$ a partir de medidas antropométricas representativas de ossos longos, como é o caso das equações de Chumlea et al.3,8, que utilizam a medida da altura do joelho. Essa medida parece sofrer poucas modificações com o envelhecimento, motivo pelo qual tem sido um dos parâmetros utilizados na estimativa da estatura ${ }^{9}$. Entretanto, é importante que a acurácia dessas equações seja testada em idosos de diferentes populações, das quais as equações não derivaram $^{10}$, confirmando, assim, sua ampla aplicabilidade ${ }^{4,11,12}$. Nesse contexto, o objetivo do presente estudo foi avaliar o uso da altura do joelho como medida alternativa confiável na avaliação nutricional antropométrica de idosos atendidos em um ambulatório de geriatria.

\section{MÉ T O D O S}

Este é um estudo transversal observacional onde foram avaliados indivíduos idosos (60 anos ou mais) durante a primeira consulta em um ambulatório de geriatria de um hospital universitário de nível terciário que atende pacientes do Sistema Único de Saúde (SUS) ${ }^{13}$. Foram excluídos idosos com algum tipo de restrição de mobilidade. O período da pesquisa foi de novembro de 2009 a novembro de 2010. As variáveis investigadas foram: gênero, idade, raça, estado civil, escolaridade, renda, peso, estatura (aferida e estimada) e altura do joelho.

Os dados demográficos foram obtidos através de entrevista estruturada. O peso foi aferido em quilogramas $(\mathrm{kg})$, em balança plataforma Welmy ${ }^{\circledast}$ (Santa Bárbara d'Oeste, São Paulo) com precisão de 0,1 kg, com o idoso no centro da base do aparelho, em posição ortostática, braço estendido em estado de relaxamento ao longo do corpo, palma da mão voltada para a coxa, sem sapatos, descartadas vestes e objetos mais pesa- dos $^{14}$. A Estatura (E1) foi aferida em metros (m), utilizando estadiômetro vertical acoplado à balança tendo o corpo erguido em extensão máxima e cabeça ereta, em posição de Frankfurt com as costas, as nádegas e a parte posterior dos joelhos encostados ao estadiômetro, os calcanhares juntos e os dedos dos pés afastados, formando um ângulo de $60^{\circ}$, aproximadamente; foram removidos quaisquer ornamentos nos cabelos ${ }^{14} \mathrm{e}$ a alça do estadiômetro foi pousada firmemente sobre a cabeça do participante. O idoso foi instruído a permanecer o mais ereto possível, respirando profundamente e mantendo a posição.

Altura do joelho foi aferida com o idoso sentado, tornozelo e joelho flexionados em ângulo reto ${ }^{3}$ e posicionando a base da régua antropométrica sob o calcanhar do pé direito e a haste pressionando a cabeça da fíbula ${ }^{15}$. A estatura Estimada (E2) através da altura do joelho foi obtida através das equações de Chumlea et al. ${ }^{3}$ segundo o gênero: homens $=[64,19-(0,04 x$ idade $)+(2,02 \times$ altura do joelho em $\mathrm{cm})$ ] e mulheres $=[84,88-(0,24 \times$ idade $)+(1,83 \times$ altura do joelho em cm)]. Já o IMC foi calculado segundo a equação de Quetelet (IMC=peso/estatura $\left.{ }^{2}\right)^{16}$ para as medidas de E1 (IMC1) e E2 (IMC2) e classificado segundo Lipschitz ${ }^{17}$.

Os dados foram analisados por meio do Statistical Package for the Social Sciences (SPSS Inc., Chicago, llinois, Estados Unidos) versão 17.0. Medidas de tendência central, dispersão e proporção foram usadas na análise descritiva dos dados. A homogeneidade entre as medidas aferidas e as estimadas foi testada por meio do Coeficiente de Correlação Intraclasse (CCI), e a concordância foi classificada segundo proposta de Fleiss \& Cohen ${ }^{18}$ : fraca $(<0,4)$; regular $(0,4-0,75)$; e excelente $(0,75-1,00)$.

A verificação da concordância entre as categorias do estado nutricional, a partir do IMC 1 e IMC2, foi realizada através do coeficiente Kappa ponderado com pesos quadráticos $\left(k w^{2}\right)^{18}$. Este avalia a homogeneidade considerando não só o grau de concordância perfeita entre as duas classificações, mas também a magnitude da discor- 
dância, ao atribuir pesos diferenciados de acordo com a maior ou menor proximidade entre as categorias da variável. O uso do Kappa ponderado está indicado quando o instrumento mede categorias ordenadas, como é o caso do $\mathrm{IMC}^{18}$. A classificação desse coeficiente foi realizada segundo Landis \& Koch $^{19}$, os quais propõem cinco categorias: ruim (0); fraca $(0,01$ a 0,20$)$; regular $(0,21$ a 0,40$)$; moderada $(0,41$ a 0,60$)$; substancial $(0,61$ a 0,80$)$; e quase-perfeita $(>0,80)^{19}$. Para a avaliação visual do padrão entre as medidas foi utilizada a disposição gráfica de Bland \& Altman ${ }^{20}$, que permite avaliar a concordância entre as variáveis, o viés, outliers e tendência ${ }^{20}$. Foram consideradas significativas as análises estatísticas com $p \geq 0,05$.

O projeto de pesquisa foi aprovado pelo Comitê de Ética em Pesquisa da Pontifícia Universidade Católica do Rio Grande do Sul, registro CEP 09/04785, e todos os pesquisados assinaram o Termo de Consentimento Livre e Esclarecido.

\section{RES U LTA DOS}

As características sociodemográficas dos indivíduos investigados estão descritas na Tabela 1. A amostra foi composta de 186 idosos, de 60 a 93 anos, sendo a faixa etária mais frequente a de 71 a 80 anos. A maior parte da amostra foi constituída de mulheres $(81,7 \%)$, de indivíduos da raça branca $(81,7 \%)$, de viúvos $(44,1 \%)$, de idosos que estudaram, mas que não completaram o primeiro grau $(61,3 \%)$ e de indivíduos com renda de mais de um a três salários mínimos $(R \$$ 510,00/mês).

$\mathrm{Na}$ Tabela 2 observa-se que o $\mathrm{CCl}$ entre as médias da E1 $(1,54 \pm 0,08 \mathrm{~m})$ e da E2 $(1,57 \pm 0,07 \mathrm{~m})$ foi de 0,84 (Intervalo de Confiança de 95\% IC95\%=0,66-0,91) ( $p<0,001)$. As médias do IMC1 e IMC2 foram, respectivamente, $28,96 \pm 5,86 \mathrm{~kg} / \mathrm{m}^{2}$ e $27,95 \pm 5,47 \mathrm{~kg} / \mathrm{m}^{2}$, e a concordância desses dados foi estatisticamente significativa $(p<0,001)$, a partir do CCI 0,96 (IC95\%=0,92-0,98).

Na Figura 1, está a representação gráfica do padrão da concordância entre as medidas de E1 e E2 (A) e IMC1 e IMC2 (B), pelo método de
Bland \& Altman ${ }^{20}$. As diferenças entre E1 e E2 foram distribuídas de acordo com a média destas duas medidas e observa-se que valores de deltas individuais oscilam em torno de zero. A média das diferenças foi $0,03 \mathrm{~m}$ (IC95\%=-0,03 a -0,02) e o limite de concordância de -0,12 m a 0,07 m. A dispersão das diferenças entre as medidas apresentou um comportamento aleatório e sistemático. Para as diferenças entre as medidas de IMC 1 e IMC2, também se observam valores de deltas individuais oscilando em torno de zero. A média das diferenças foi de $1,01 \mathrm{~kg} / \mathrm{m}^{2}$ (IC95\% $=0,74$ a 1,29$)$ e o limite de concordância de $-2,70$ a $4,73 \mathrm{~kg} / \mathrm{m}^{2}$.

A classificação do estado nutricional e a avaliação da concordância entre as observações estão descritas na Tabela 3. Entre os idosos ava-

Tabela 1. Características sociodemográficas dos idosos atendidos em ambulatório de geriatria de um hospital universitário, de novembro/2009 a novembro/2010 ( $\mathrm{N}=186)$.

\begin{tabular}{|c|c|c|}
\hline Dados sociodemográficos & $\mathrm{N}$ & $\%$ \\
\hline \multicolumn{3}{|l|}{ Sexo } \\
\hline Feminino & 152 & 81 \\
\hline Idade (anos - M $\pm D P$ ) & 74,3 & \pm 7 \\
\hline \multicolumn{3}{|l|}{ Faixa etária } \\
\hline 60 a 70 anos & 54 & 29 \\
\hline 71 a 80 anos & 98 & 52 \\
\hline 81 a 100 anos & 34 & 18 \\
\hline \multicolumn{3}{|l|}{ Raça } \\
\hline Branca & 152 & 81 \\
\hline Negra & 20 & 10 \\
\hline Parda & 14 & 7 \\
\hline \multicolumn{3}{|l|}{ Estado civil } \\
\hline Casado & 63 & 33 \\
\hline Solteiro & 14 & 7 \\
\hline Separado ou divorciado & 27 & 14 \\
\hline Viúvo & 82 & 44 \\
\hline \multicolumn{3}{|l|}{ Escolaridade } \\
\hline Nunca estudou & 23 & 12 \\
\hline Estudou mas não completou o $1^{\circ} \mathrm{grau}$ & 114 & 61 \\
\hline Completou o $1^{\circ} \mathrm{grau}$ & 49 & 26 \\
\hline \multicolumn{3}{|l|}{ Renda } \\
\hline Até 1 salário mínimo & 72 & 38 \\
\hline >1 a 3 salários mínimos & 95 & 51 \\
\hline >3 salários mínimos & 19 & 10 \\
\hline
\end{tabular}

Nota: Salário mínimo de R\$510,00/mês.

M: Média; DP: Desvio-Padrão 
liados houve uma prevalência de excesso de peso de $59,7 \%$ para o IMC1 e de 53,2\% para o IMC2. O índice Kappa ponderado com pesos quadráticos comparando o estado nutricional determinado a partir do IMC1 com o do IMC2 foi de 0,79 (IC95\%=0,70 a 0,88).

Tabela 2. Valores mínimo, máximo, média, Desvio-Padrão (DP) e Coeficiente de Correlação Intraclasse (CCI) das estaturas e Índices de Massa Corporal (IMC), aferidos e estimados, de idosos atendidos em ambulatório de geriatria de um hospital universitário, de e novembro/2009 a novembro/2010 ( $N=186)$.

\begin{tabular}{lcccccc}
\hline Medidas & Mínimo & Máximo & Média & DP & CCI (IC95\%) & $p$ \\
\hline Estatura aferida $(\mathrm{m})$ & 1,38 & 1,78 & 1,54 & 0,08 & \multirow{2}{*}{$0,84(0,66-0,91)$} & $<0,001$ \\
Estatura estimada $(\mathrm{m})$ & 1,57 & 1,76 & 1,57 & 0,07 & & \\
IMC aferido $\left(\mathrm{kg} / \mathrm{m}^{2}\right)$ & 17,27 & 49,15 & 28,96 & 5,86 & & \\
IMC estimado $\left(\mathrm{kg} / \mathrm{m}^{2}\right)$ & 17,04 & 45,97 & 27,95 & 5,47 & $0,92-0,98)$ & $<0,001$ \\
\hline
\end{tabular}

Nota: IC95\%: Intervalo de Confiança de 95\%.
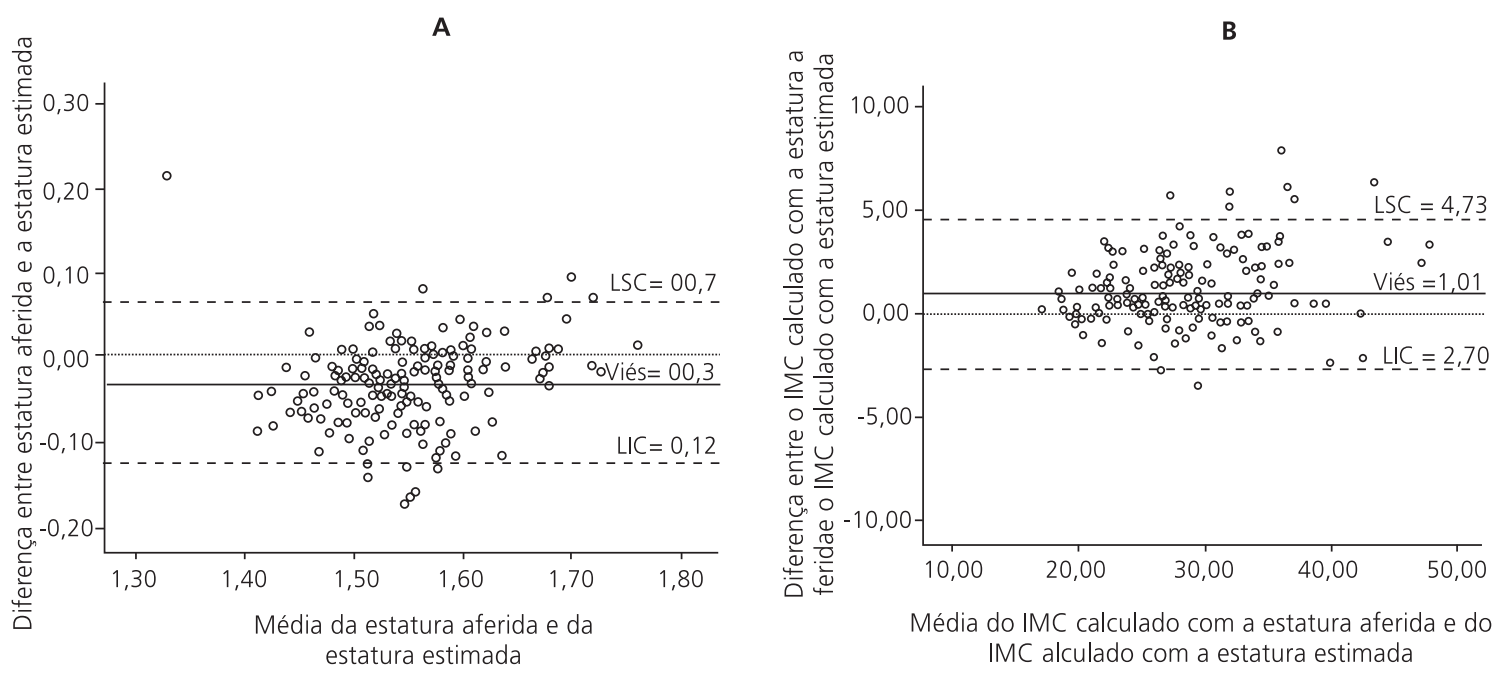

Figura 1. Gráficos Bland \& Altman ${ }^{20}$ da comparação da aproximação da estatura aferida com a estatura estimada (A) e do Índice de Massa Corporal (IMC) com estatura aferida e do IMC com estatura estimada (B). Ambulatório de hospital universitário, de novembro/2009 a novem-bro/2010.

Nota: LSC: Limite Superior de Concordância; LIC: Limite Inferior de Concordância.

Tabela 3. Proporção de concordâncias (acordos observados) entre o estado nutricional, classificado através do índice de massa corporal obtido a partir da estatura aferida e da estatura estimada, e o Kappa ponderado de idosos atendidos em ambulatório de geriatria de um hospital universitário, de novembro/2009 a novembro/2010 ( $N=186)$.

\begin{tabular}{|c|c|c|c|c|c|c|c|c|}
\hline \multirow{3}{*}{ Classificação do IMC2 } & \multicolumn{6}{|c|}{ Classificação do IMC1 } & \multirow{2}{*}{\multicolumn{2}{|c|}{ Total }} \\
\hline & \multicolumn{2}{|c|}{ Magreza } & \multicolumn{2}{|c|}{ Eutrofia } & \multicolumn{2}{|c|}{ Sobrepeso } & & \\
\hline & $n$ & $\%$ & $\mathrm{n}$ & $\%$ & $n$ & $\%$ & $\mathrm{n}$ & $\%$ \\
\hline Magreza & 16 & 8,6 & 1 & 0,5 & 0 & 0,0 & 17 & 9,1 \\
\hline Eutrofia & 12 & 6,5 & 39 & 21,0 & 7 & 3,8 & 58 & 31,2 \\
\hline Sobrepeso & 0 & 0,0 & 19 & 10,2 & 92 & 49,5 & 111 & 59,7 \\
\hline Total & 28 & 15,1 & 59 & 31,7 & 99 & 53,2 & 186 & 100,0 \\
\hline
\end{tabular}

Nota: IMC1: Índice de Massa Corporal calculado a partir da estatura aferida; IMC2: Calculado a partir da estatura estimada; Kappa ponderado com pesos quadráticos $\left(\mathrm{kW}^{2}\right)$ : 0,79 Intervalo de Confiança 95\% (IC95\%=0,70-0,88). 


\section{I S C U S S Ã O}

O trabalho de Trotter \& Gleser ${ }^{21}$, publicado há mais de seis décadas, demonstra que estimar estatura a partir de ossos longos vem sendo objeto de estudos há muito tempo e, nesse período, várias equações estimativas foram desenvolvidas a partir da medida da altura do joelho $3,4,8,22-24$. O que tem possibilitado o uso dessa medida para estimar a estatura de idosos, tanto em países

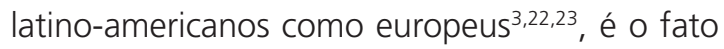
de que o comprimento da altura do joelho parece se manter inalterado da vida adulta até o envelhecimento.

Diversas equações foram desenvolvidas, de acordo com sexo e etnia, a partir de determinadas populações e, por vezes, seu uso se mostrou ineficaz em amostras diferentes daquelas das quais se originaram, como demonstraram Mendoza-Núnez et al. ${ }^{24}$. Entretanto, as equações de Chumlea et al. ${ }^{3,8}$ continuam sendo as mais aceitas em países ocidentais e as que as sucederam parecem trazer resultados similares ${ }^{25}$.

Assim, na escolha da equação para estimar a estatura, dentre as diversas existentes, foi considerado importante conhecer as características étnicas da população da qual ela se originou. Aquelas desenvolvidas por Chumlea et al. ${ }^{3}$ foram escolhidas por derivarem de uma amostra caucasiana, divisão étnica que inclui descendentes de europeus, com pele variando entre as cores clara e morena. Essas características podem ser encontradas na população idosa do nosso estado, a qual apresenta enorme heterogeneidade étnica devido, principalmente, à colonização por açorianos, alemães e italianos ${ }^{26}$. Além disso, as medidas médias da altura do joelho aferidas neste estudo $(51,8 \pm 2,4 \mathrm{~cm}$ e $48,1 \pm 2,7 \mathrm{~cm}$, respectivamente para homens e mulheres) estão inseridas entre os valores médios obtidos na população usada para desenvolver a equação de Chumlea et al. ${ }^{3}$ $(53,3 \pm 2,7 \mathrm{~cm}$ nos homens e $49,4 \pm 2,3 \mathrm{~cm}$ nas mulheres), aspecto que reforça a escolha dessas equações.
Entre as características encontradas na amostra estudada, registramos a maior frequência de mulheres $(81,7 \%)$, que pode ser explicada pelo fato de as mesmas buscarem, mais do que os homens, realizar acompanhamento médico contínuo ${ }^{27}$. Ademais, devido à desigualdade de gênero na expectativa de vida, que resulta em um maior número de mulheres do que de homens entre os idosos ${ }^{28}$.

A diferença entre as médias da E1 $(1,54 \pm 0,08 \mathrm{~m})$ e da $\mathrm{E} 2(1,57 \pm 0,07 \mathrm{~m})$ a partir do $\mathrm{CCl}$ demonstram concordância excelente $(0,84)$ ou, no mínimo, satisfatória, considerando o limite inferior do intervalo de confiança do $\mathrm{CCl}(0,66)$. Esse dado sugere que a variação entre as medidas foi pequena ${ }^{18}$. Complementarmente, na representação gráfica de Bland \& Altman ${ }^{20}$, a oscilação das diferenças das médias em torno de zero indica medidas semelhantes. A média das diferenças $(0,03 \mathrm{~m})$ e o limite de concordância demonstram que as medidas se aproximam, uma vez que o viés é próximo de zero e os limites apontam para diferenças menores do que 0,12 m entre as duas medidas, valor considerado aceitável. A reta paralela ao eixo x, 0,03m abaixo da linha de diferença igual a zero, indica um viés fixo da medida e leve tendência de superestimação de E2 em relação à E1. O comportamento aleatório da dispersão das diferenças entre as medidas, com pouca discrepância fora dos limites de concordância, é outro aspecto positivo da análise.

Várias pesquisas avaliando equações preditivas foram encontradas nas bases de dados, entretanto, os resultados somente serão comparados com aqueles produzidos a partir das equações desenvolvidas por Chumlea et al. ${ }^{3}$. Ao confrontar-se estatura aferida e estimada dois estudos, avaliando indivíduos de origem asiática, obtiveram conclusões consistentes entre si. Myers et al. ${ }^{29}$, em 1994, validaram o uso da equação de Chumlea et al. ${ }^{3}$ em 32 idosos nipo-americanos atendidos em ambulatório hospitalar e concluíram que a estatura estimada para aquela amostra foi significativamente diferente daquelas para cauca- 
sianos $^{29}$. Assim, também Li et al. ${ }^{30}$ em 2000, realizaram estudo similar com 47 idosos de descendência chinesa, pacientes de um ambulatório ou residentes de albergue para idosos e consideraram que são necessários modelos específicos para as diferentes etnias ${ }^{30}$.

Em idosos europeus doentes a utilização da medida da altura do joelho foi considerada com suficiente acurácia e razoável precisão para estimar a estatura, como verificado por Berger et al. ${ }^{31}$ em 2008 que estudou amostra de 250 idosos pacientes críticos de hospital universitário na Suíça. Cereda et al.32, em 2007, consideraram viável a utilização da estatura estimada através da altura do joelho de 231 idosos italianos residentes em instituições de longa permanência, na determinação do Geriatric Nutritional Risk Index, e indiretamente validaram a equação de Chumlea et al. ${ }^{3}$.

Shahar \& Pooy ${ }^{12}$ alertaram para a possibilidade de que equações derivadas de populações de estatura mais elevada, como os caucasianos, possam ser menos precisas quando aplicadas em grupos de menor estatura. Essa indicação pode ser observada nos estudos analisados neste trabalho.

No que diz respeito à população brasileira, pesquisas que verificaram a aplicabilidade das fórmulas de estimativa de estatura para idosos da região Nordeste obtiveram dados discrepantes. Foi observada diferença significativa entre as medidas de estatura aferida e estimada de 64 pacientes idosos de um hospital de Fortaleza (CE), avaliados em $2002^{33}$. Já entre 30 idosas matriculadas em uma academia de João Pessoa (PB), em $2007^{34}$, os dados foram concordantes.

Na região Sudeste, estudo realizado em 2008 com 344 idosos institucionalizados do Rio de Janeiro também chegou à mesma conclusão ${ }^{35}$. Entretanto, trabalho desenvolvido na cidade de Porto Alegre (RS) em 2013 com 131 idosos residentes de instituições de longa permanência não obteve os mesmos resultados; houve diferença significativa entre a medida da estatura aferida e a estimada ${ }^{36}$.
Em pesquisa recente, autores avaliaram 547 idosos da comunidade de Viçosa (MG), e constataram que a estatura estimada foi superior à estatura aferida, especialmente nas mulheres, produzindo um IMC inferior. Além disso, demonstraram preocupação em relação ao uso dessas equações em determinados grupos da população ${ }^{37}$. Estudos anteriores, que utilizaram o mesmo método da presente pesquisa, sinalizam um intervalo de concordância entre $\pm 8-9 \mathrm{~cm}$ entre as medidas aferidas e estimadas $3,8,25$.

Em relação à média do $\mathrm{IMC} 1(28,96 \pm 5,86$ $\left.\mathrm{kg} / \mathrm{m}^{2}\right)$ e do IMC2 $\left(27,95 \pm 5,47 \mathrm{~kg} / \mathrm{m}^{2}\right)$, a concordância encontrada neste estudo foi considerada excelente ${ }^{18}(\mathrm{CCl}=0,96)$, mesmo tendo em vista o limite inferior do intervalo de confiança $(\mathrm{CCl}=0,92)$ sugerindo uma oscilação pequena entre as variáveis. Para esses resultados, a classificação do estado nutricional mostrou uma prevalência de sobrepeso de 59,7\% para o IMC1 e de 53,2\% para o IMC2, segundo Lipschitz ${ }^{17}$.

Na verificação gráfica do comportamento das diferenças entre as medidas de IMC1 e IMC2 (média=1,01kg/m², IC95\%=0,74 a 1,29kg/m²), observam-se valores de deltas individuais oscilando em torno de zero, indicando medidas semelhantes. O limite de concordância sinaliza uma aproximação razoável entre as medidas, uma vez que o viés é próximo de zero e os limites apontam para diferenças inferiores a $4,73 \mathrm{~kg} / \mathrm{m}^{2}$, entre as mesmas, valores que podem ser um pouco elevados tratando-se de IMC. Como seria de esperar, a reta paralela ao eixo $x, 1,01 \mathrm{~kg} / \mathrm{m}^{2}$ acima da linha de diferença igual a zero, indica um viés fixo da medida e a tendência de subestimação do IMC2 em relação ao IMC1, uma vez que o IMC foi calculado com uma medida de estatura superestimada resultando em um valor menor. Quanto ao comportamento das variáveis, pode-se verificar que a maioria das medidas está dentro do intervalo, de forma aleatória; entretanto, observa-se que os obesos saem um pouco da faixa.

Os limites de concordância observados nos gráficos de Bland \& Altman ${ }^{20}$ precisam ser ana- 
lisados quanto aos possíveis impactos na utilização da medida da estatura estimada na avaliação nutricional dos idosos através do IMC. Considerados a estatura $(1,63 \mathrm{~m})$ e o peso $(70 \mathrm{~kg})$ médios dos idosos do Rio Grande do Sul28, e aplicados os resultados encontrados neste estudo, o limite de concordância inferior $(-0,12 \mathrm{~m})$ produzirá um IMC superestimado em até $4,35 \mathrm{~kg} / \mathrm{m}^{2}$, e o limite de concordância superior $(0,07 \mathrm{~m})$ poderá resultar em um IMC subestimado em até $-2,13 \mathrm{~kg} / \mathrm{m}^{2}$. Fried et al. ${ }^{38}$ encontraram um IMC 2,5 kg/m² menor e $1,0 \mathrm{~kg} / \mathrm{m}^{2}$ maior. O índice Kappa ponderado com pesos quadráticos, comparando o estado nutricional determinado a partir do IMC1 e do IMC2 (0,79; IC95\%=0,70 a 0,88), mostrou concordância entre substancial e quase perfeita ou, pelo menos, boa, considerando o limite inferior do intervalo de confiança $(0,70)^{19}$.

$\mathrm{Na}$ busca de comparativos na literatura internacional foi possível contar com Ritz et al. ${ }^{39}$ os quais consideraram que a estatura estimada produziu mínimo viés e sugeriram propagação de mínimo erro para o IMC. A diferença entre os IMC foi de $0,27 \mathrm{~kg} / \mathrm{m}^{2}$ e os autores consideram que o uso da altura do joelho quase não afeta o cálculo do mesmo. Além desse estudo, também destaca-se Cereda et al. ${ }^{32}$, que encontraram uma diferença não significativa entre o IMC produzido com estatura aferida e o obtido com a estimada.

Nos trabalhos avaliando idosos brasileiros foram encontradas duas pesquisas com resultados contraditórios. Em idosos hospitalizados de Fortaleza (CE), foi observado que a diferença significativa encontrada nas estaturas repercutiu no $\mathrm{IMC}^{33}$. Porém, a avaliação de idosos institucionalizados do Rio de Janeiro indicou que a estatura estimada a partir da medida da altura do joelho aproximou-se do valor real da estatura, possibilitando seu uso na estimativa do IMC quando da avaliação do estado nutricional de idosos. Esse resultado deveu-se à alta concordância encontrada entre os IMC e a um Kappa ponderado entre o IMC aferido e o estimado de $0,78^{35}$.

Finalmente, apontam-se pontos fortes e limitações no presente estudo. Pelo menos três fatores positivos merecem ser destacados: a) a temática do estudo, uma vez que se buscou investigar a aplicabilidade das equações preditivas de estatura de Chumlea et al. 3,8 em uma população específica, ampliando o conhecimento que já vem sendo demonstrado em grupos diversos por pesquisadores nacionais e internacionais; b) a abordagem estatística escolhida com a utilização de testes robustos; c) a obtenção de todas as medidas antropométricas por uma única nutricionista, treinada e certificada pela International Society for the Advancement of Kinanthropometry4, eliminando o viés interavaliadores. Entre as limitações do estudo, destaca-se a restrição dos resultados a indivíduos idosos com características e condições similares (idosos atendidos em um ambulatório geriátrico de referência - atenção terciária).

Nesse sentido, a heterogeneidade étnica da população brasileira, por si só, incita a questionamentos quanto à aplicabilidade das equações preditivas de forma generalizada, sugerindo a necessidade de avaliá-las em diversos segmentos regionais. Os resultados aqui observados, de pequena variabilidade entre as medidas aferidas e estimadas (principalmente da estatura), corroborando resultados previamente publicados na literatura nacional e internacional, podem estimular a realização de estudos com amostras maiores e representativas das diferenças étnicas encontradas no Brasil, bem como em diferentes cenários da saúde brasileira, especialmente na atenção básica à saúde.

\section{O N CLUS Ã O}

Os resultados do presente estudo mostram que a altura do joelho é uma medida alternativa confiável na avaliação nutricional de idosos, sendo possível utilizá-la como estimativa da estatura para cálculo do IMC em indivíduos atendidos em ambulatório de geriatria. Essa alternativa é especialmente útil para aqueles que apresentam limitações para a aferição das medidas antropométricas, como os indivíduos com instabilidade postural e restrição de mobilidade. 


\section{COLABORADORES}

VE CLOSS colaborou na concepção, desenho, coleta, análise, interpretação dos dados do estudo e elaboração do manuscrito. AMP FEOLI colaborou na concepção do estudo e revisão do manuscrito. CHA SCHWANKE colaborou na concepção do estudo, supervisão da coleta dos dados e revisão do manuscrito.

\section{REFERÊ NCIAS}

1. World Health Organization. Physical status: The use and interpretation of anthropometry. Report of a WHO Expert Committee. Geneva: World Health Organization; 1995.

2. Pereira $C A$. Avaliação nutricional na terceira idade. In: Magnoni D, Cukier C, Oliveira PA, editores. Nutrição na terceira idade. $2^{a}$ ed. São Paulo: Sarvier; 2010. p.20-36.

3. Chumlea WC, Roche AF, Steinbaugh ML. Estimating stature from knee height for persons 60 to 90 years of age. J Am Geriatr Soc. 1985; 33(2):116-20. http:// dx.doi.org/10.1111/j.1532-5415.1985.tb02276.x

4. Dunsky A, Zach S, Zeev A, Goldbourt U, Shimony T, Goldsmith R, et al. Prediction of standing height among Israeli older adults: Results from a national survey. Ann Hum Biol. 2012; 39(6):499-504. http:// dx.doi.org/10.3109/03014460.2012.718795

5. Miranda EGA, Camargo LRB, Costa TMB, Pereira RCG. Manual de avaliação nutricional do adulto e do idoso. Rio de Janeiro: Rubio; 2012.

6. Cintra MTG, Bicalho MAC. Modificações fisiológicas no envelhecimento. In: Campos ACV, Berlezi EM, Correa AHM, editores. Envelhecimento: um processo multidimensional. Ijuí: Unijuí; 2014. p.125-48.

7. Lorini C, Collini F, Castagnoli M, Di Bari M, Cavallini MC, Zaffarana N, et al. Using alternative or direct anthropometric measurements to assess risk for malnutrition in nursing homes. Nutrition. 2014; 30(10):1171-6. http://dx.doi.org/10.1016/j.nut. 2014.03.005

8. Chumlea WC, Guo SS, Wholihan K, Cockram D, Kuczmarski RJ, Johnson CL. Stature prediction equations for elderly non-hispanic white, non-hispanic black, and Mexican-American persons developed from NHANES III Data. J Am Diet Assoc. 1998; 98(2):137-42.

9. Perissinotto E, Pisent C, Sergi G, Grigoletto F, Enzi $G$. Anthropometric measurements in the elderly: Age and gender differences. Br J Nutr. 2007; 87(2):177. http://dx.doi.org/10.1079/BJN2001487
10. Mitchell CO, Lipschitz MD. Detection of proteincalorie malnutrition in the elderly. Am J Clin Nutr. 1982; 35(2):398-406.

11. Dock-Nascimento DB, Aguilar-Nascimento JE, Costa HCBAL, Vale HVV, Gava MM. Precisão de métodos de estimativa do peso e altura na avaliação do estado nutricional de pacientes com câncer. Rev Bras Nutr Clín. 2005; 21(2):111-6.

12. Shahar S, Pooy NS. Predictive equations for estimation of stature in Malaysian elderly people. Asia Pac J Clin Nutr. 2003; 12(1):80-4.

13. Macinko J, MHarris MJ, Phil D. Brazil's family health strategy: Delivering community-based primary care in a universal health system. New Eng J Med. 2015; 372(23):2177-81. http://dx.doi.org/10.1056/ NEJMp1501140

14. Centers for Disease Control and Prevention, National Health and Nutrition Examination Survey. Anthropometry procedures manual. Atlanta (GA): Centers for Disease Control and Prevention; 2007 [cited 2014 Aug 29]. Available from: http://www. cdc.gov/nchs/data/nhanes/nhanes_07_08/manual_ an.pdf

15. Najas MS, Sachs A. Avaliação nutricional do idoso. In: Papaléo Netto M, editor. Gerontologia. São Paulo: Atheneu; 2005. p.242-7.

16. Garrow JS, Webster J. Quetelet's index (W/H2) as a measure of fatness. Int J Obes. 1985; 9(2):147-53.

17. Lipschitz DA. Screening for nutritional status in the elderly. Prim Care. 1994; 21(1):55-67.

18. Fleiss $\mathrm{JL}$, Cohen J. The equivalence of weighted Kappa and the intraclass correlation coeficient as a measures of reliability. Education Psychol Measurem. 1973; 33(3):613-9. http://dx.doi.org/10.1177/0 01316447303300309

19. Landis JR, Koch GG. The measurement of observer agreement for categorical data. Biometrics. 1977; 33(1):159-74.

20. Bland JM, Altman DG. Statistical methods for assessing agreement between two methods of clinical measurement. Lancet. 1986; 1(8476):307-10. http://dx.doi.org/10.1016/S0140-6736(86)90837-8

21. Trotter M, Gleser GC. Estimation of stature from long bones of American Whites and Negroes. Am J Phys Anthropol. 1952; 10(4):463-514. http:// dx.doi.org/10.1002/ajpa.1330100407

22. Donini LM, Felice MR, Bernardini L, Ferrar G, Rossano A, Medici $M$, et al. Prediction of stature in the Italian elderly. J Nutr Health Aging. 2000; 4(2):72-6.

23. Lera L, Luis Santos J, Garcia C, Arroyo P, Albala C. Predictive equations for stature in the elderly: $A$ study in three Latin American cities. Ann Hum Biol. 2005; 32(6):773-81. http://dx.doi.org/10.1080/ 03014460500421304 
24. Mendoza-Núnez VM, Sánchez-Rodriguez MA, Cervantes-Sandoval A, Correa-Munoz E, Vargas-Guadarrama LA. Equations for predicting height for elderly Mexican Americans are not applicable for elderly Mexicans. Am J Hum Biol. 2002; 14(3):351-5. http://dx.doi.org/10.1002/ajhb.10029

25. Cereda E, Bertoli S, Vanotti A, Battezzati A. Estimated height from knee-height in Caucasian elderly- implications on nutritional status by mini nutritional assessment. J Nutr Health Aging. 2010; 14(1):16-22. http://dx.doi.org/10.1007/s12603-01 0-0004-2

26. Gottlieb MGV, Schwanke CHA, Gomes I, Cruz IBM. Envelhecimento e longevidade no Rio Grande do Sul: um perfil histórico, étnico e de morbimortalidade dos idosos. Rev Bras Geriat Gerontol. 2011; 14(2):365-80. http://dx.doi.org/10.1590/\$1809-98 232011000200016

27. Gomes R, Nascimento EF, Araújo FC. Por que os homens buscam menos os serviços de saúde do que as mulheres? As explicações de homens com baixa escolaridade e homens com ensino superior. Cad Saúde Pública. 2007; 23(3):565-74. http:// dx.doi.org/10.1590/S0102-311X2007000300015

28. Instituto Brasileiro de Geografia e Estatística. Censos demográficos. Brasília: IBGE; 2013 [acesso 2015 jan 12]. Disponível em: http://www.ibge.gov.br

29. Myers SA, Takiguchi RN, Yu M. Stature estimated from knee height in elderly Japanese Americans. J Am Geriatr Soc. 1994; 42(2):157-60. http://dx. doi.org/10.1111/j.1532-5415.1994.tb04945.x

30. Li ETS, Tang EKY, Wong CYM, Lui SSH, Chan VYN, Dai DLK. Predicting stature from knee height in Chinese elderly subjects. Asia Pac J Clin Nutr. 2000; 9(4):252-5. http://dx.doi.org/10.1046/j. 1440-60 47.2000.00158.x

31. Berger MM, Cayeux MC, Schaller MD, Soguel L, Piazza G, Chioléro RL. Stature estimation using the knee height determination in critically ill patients 2008. e-SPEN J. 2008; 3(2):e84-e8. http://dx.doi. org/10.1016/j.eclnm.2008.01.004

32. Cereda E, Limonta D, Pusani C, Vanotti A. Feasible use of estimated height for predicting outcome by the geriatric nutritional risk index in long-term care resident elderly. Gerontology. 2007; 53(4):184-6. http://dx.doi.org/10.1159/000099468

33. Sampaio HAC, Melo MLP, Almeida PC, Benevides $A B$. Aplicabilidade das fórmulas de estimativa de peso e altura para idosos e adultos. Rev Bras Nutr Clín. 2002; 17(4):117-21.

34. de Oliveira L. Estatura e massa corporal mensurados e preditos através das equações de Chumlea em idosas. Fit Perform J. 2007; 6(3):152-5. http:// dx.doi.org/10.3900/fpj.6.3.152.p

35. Amorin RB, Santa Cruz MAC, Souza-Júnior PRB, Mota JC, González C. Medidas de estimación de la estatura aplicadas al Índice de Masa Corporal (IMC) en la evaluación del estado nutricional de adultos mayores. Rev Chil Nutr. 2008; 35(Supl. 1):272-9. http://dx.doi.org/10.4067/S0717-75182008 000400003

36. Souza R, Fraga JS, Gottschall CBA, Busnello FM, Rabito El. Avaliação antropométrica em idosos estimativas de peso e altura e concordância entre classificações de IMC. Rev Bras Geriatr Gerontol. 2013; 16(1):81-90. http://dx.doi.org/10.1590/S1 809-98232013000100009

37. Fogal AS, Franceschini SC, Priore SE, Cotta RM, Ribeiro AQ. Stature estimation using the knee height measurement amongst Brazilian elderly. Nutr Hosp. 2014; 31(2):829-34. http://dx.doi.org/10.3 305/nh.2015.31.2.7618

38. Fried H, Adolfsson ET, Rosenblad A, Nydahl M. Agreement between different methods of measuring height in elderly patients. J Hum Nutr Diet. 2013;26(5):504-11. http://dx.doi.org/10.11 11/jhn. 12031

39. Ritz P. Validity of measuring knee-height as an estimate of height in diseased French elderly persons. J Nutr Health Aging. 2004; 8(5):386-8.

40. Marfell-Jones M, Olds T, Stewart A, Carter L. International standars for anthropometric assessment. Potchefstroom: International Society for the Advancement of Kinanthropometry; 2006.

Recebido: fevereiro 5, 2015 Versão final: junho 23, 2015 Aprovado: julho 8, 2015 